\title{
Role of Cholinergic Anti-Inflammatory Pathway in Treatment of Intestinal Ischemia-Reperfusion Injury by Electroacupuncture at Zusanli
}

\author{
Yanxia Geng, ${ }^{1}$ Dong Chen, ${ }^{2}$ Jiang Zhou, ${ }^{1}$ Hua Jiang, ${ }^{1}$ and Haidong Zhang ${ }^{1}$ \\ ${ }^{1}$ Intensive Care Unit, Affiliated Hospital of Nanjing University of Traditional Chinese Medicine, 155 Hanzhong Road, \\ Nanjing 210029, China \\ ${ }^{2}$ Acupuncture and Rehabilitation Department, Affiliated Hospital of Nanjing University of Traditional Chinese Medicine, \\ 155 Hanzhong Road, Nanjing 210029, China
}

Correspondence should be addressed to Dong Chen; xiaodo945@sina.com

Received 26 May 2017; Revised 17 July 2017; Accepted 29 October 2017; Published 3 December 2017

Academic Editor: Manel Santafe

Copyright (C) 2017 Yanxia Geng et al. This is an open access article distributed under the Creative Commons Attribution License, which permits unrestricted use, distribution, and reproduction in any medium, provided the original work is properly cited.

\begin{abstract}
Electroacupuncture (EA) at Zusanli is a widely used method for the treatment of intestinal ischemic disease. The current study attempts to investigate the possible mechanism from the point of cholinergic anti-inflammatory pathway (CAP) in rats. Thirty rats were divided into five groups: control group, I/R group, EA group (I/R $+\mathrm{EA}), \mathrm{PNU}$ group (I/R $+\alpha 7 \mathrm{nAChR}$ agonist), and $\alpha$-BGT group (I/R $+\mathrm{EA}+\alpha 7 \mathrm{nAChR}$ antagonist). EA and medicine injection were performed immediately after ischemia. After $2 \mathrm{~h}$ of reperfusion, blood and intestine samples were collected and intestinal histopathological score, mRNA expression of mucosal $\alpha 7$ nAChR and NF- $\kappa$ Bp65, and serum cytokine levels (IL-6, TNF- $\alpha$ ) were examined. Compared with the I/R group, the EA group and PNU group could significantly attenuate the mucosal damage, promote $\alpha 7 \mathrm{nAChR}$ mRNA expression, and reduce levels of NF- $\kappa$ Bp 65 , IL-6, and TNF- $\alpha$. Compared with the EA group, $\alpha 7$ nAChR mRNA was decreased, while concentrations of NF- $\kappa$ Bp65, IL-6, and TNF- $\alpha$ increased in the $\alpha$-BGT group. EA at Zusanli could inhibit NF- $\kappa$ Bp 65 and proinflammatory cytokines production after intestinal I/R injury; its mechanism may be related to the cholinergic anti-inflammatory pathway.
\end{abstract}

\section{Background}

Intestinal ischemic disease is a common clinical condition with limited treatment options. The Chinese medicine of electroacupuncture (EA) therapy has opened up a new field for this disease. Our previous study has shown that EA at Zusanli could reduce the expression of inflammatory cytokines, alleviate intestinal hyperpermeability, and promote repair after intestinal ischemia-reperfusion (I/R) injury [1]. However, the specific mechanism has not yet been clarified. In recent years, the cholinergic anti-inflammatory pathway (CAP) has been investigated a lot in the treatment of EA in intestinal inflammatory diseases [2], which might also help to illuminate the mechanism of ischemic disease, while ischemia is essentially an inflammatory process as well. The purpose of this study is to observe the effect of EA at Zusanli on the inflammatory response in rats' small intestine $\mathrm{I} / \mathrm{R}$ injury and reveal its relationship with CAP, so as to provide a theoretical basis for clinical treatment.

\section{Methods}

2.1. Animal Grouping. Intestinal I/R injury was carried out by superior mesenteric artery (SMA) occlusion for $30 \mathrm{~min}$ and release for 2 hours. Thirty male Sprague-Dawley rats weighing about 200-250 g were randomly divided into five groups: control group (Con), rats subjected to laparotomy with no clamping of SMA; I/R group, rats that underwent I/R injury; electroacupuncture group (EA), rats with $\mathrm{I} / \mathrm{R}$ injury with EA applied (HANS/LH202H, $2 \mathrm{~mA}, 2-100 \mathrm{~Hz}$ ) at bilateral Zusanli points (ST36 points) for $30 \mathrm{~min}$, immediately after the ischemic period started; PNU282987 group (PNU), rats with I/R injury with PNU282987 (5 $\mu \mathrm{g} / \mathrm{kg}$, Sigma) 
intraperitoneally injected immediately after ischemia started; $\alpha$-bungarotoxin group ( $\alpha$-BGT), rats with I/R injury with $\alpha$ bungarotoxin $(1 \mu \mathrm{g} / \mathrm{kg}$, Sigma) intraperitoneally injected and EA applied simultaneously immediately after ischemia.

2.2. Histopathological Examination. Rats in each group were sacrificed at $2 \mathrm{~h}$ of reperfusion. $1 \mathrm{~cm}$ of the distal ileum was removed, rinsed, and fixed in $10 \%$ formaldehyde for $\mathrm{H} \& \mathrm{E}$ staining. Histological damage was assessed by Chiu's score under a light microscope.

2.3. RT-PCR Tests of $\alpha 7 n A C h R$ and NF- $\kappa$ Bp65. Take $10 \mathrm{~cm}$ of the distal part of the small intestine, scrap off the mucosa, and grind it into powder in liquid nitrogen. Total RNA was extracted from the small intestine by TRIzol method (TransZol Up, TransGen). The transcription and amplification reaction were carried out according to the kit instructions (Thermo-Script RT-PCR System, Invitrogen). The primer sequences were as follows: $\alpha 7$ nAChR, 5' -ATCTGGGCATTGCCAGTATC-3' (sense) and $5^{\prime}$-TCCCATGAGATCCCATTCTC- $3^{\prime}$ (antisense); NFкBp65, 5' -CACAGATACCACTAAGACGCACC- $3^{\prime}$ (sense) and $5^{\prime}$-GACCGCATTCAAGTCATAGTCC- $3^{\prime}$ (antisense); $\beta$-actin, $5^{\prime}$-TCAGGTCATCACTATCGGCAAT- ${ }^{\prime}$ (sense) and $5^{\prime}$-AAAGAAAGGGTGTAAAACGCA-3' (antisense). The expected length of the amplified fragments was $199 \mathrm{bp}$ for $\alpha 7 \mathrm{nAChR}, 173 \mathrm{bp}$ for NF-кBp65, and $432 \mathrm{bp}$ for $\beta$-actin.

2.4. ELISA Tests of IL- 6 and TNF- $\alpha$. Collect $2 \mathrm{ml}$ of blood from the portal vein and store the supernatant serum at $-80^{\circ} \mathrm{C}$ after centrifugation. At the time of experiment, serum samples were thawed and ELISA tests of IL- 6 and TNF- $\alpha$ were carried out according to the manufacturer's instructions (R\&D Systems). Samples were incubated with biotin-labeled antibodies, streptavidin-HRP, substrates, and stop solution successively. The optical density (OD) value of each well was measured using a microplate reader. Standard curve was prepared according to the standard solution and corresponding OD value, and thus concentrations of IL- 6 and TNF- $\alpha$ of each sample could be calculated.

2.5. Statistical Analysis. Data were analyzed by SPSS18.0 software. All values were expressed as mean \pm standard deviation. One-way ANOVA was used to compare the data between groups. A $p$ value of $<0.05$ was considered statistically significant.

\section{Results}

3.1. Histopathology Score of Small Intestine. As Table 1 shows, Chiu's score showed significantly more severe damage of the small intestine mucosa than that in the control group $(3.7 \pm 0.5$ versus $0.3 \pm 0.5, p<0.01$ ) after I/R injury. When compared with the I/R group, the histological destruction in EA and PNU groups was much relieved ( $p=0.004$ and 0.045 , resp.). Chiu's score of the $\alpha$-BGT group did not show a significant difference from the I/R group, but it was much higher than in the EA group $(p=0.014)$, indicating that $\alpha 7 \mathrm{nAChR}$ antagonist might reverse the efficacy of EA to some extent.
TABLE 1: Chiu's scoring in each group.

\begin{tabular}{lc}
\hline Groups & Chiu's score \\
\hline Control & $0.3 \pm 0.5$ \\
I/R group & $3.7 \pm 0.5$ \\
EA group & $2.7 \pm 0.5^{*}$ \\
PNU group & $3.0 \pm 0.6^{*}$ \\
$\alpha$-BGT group & $3.5 \pm 0.6^{\triangle}$ \\
\hline
\end{tabular}

${ }^{*} p<0.05$ compared with the I/R group; ${ }^{\triangle} p<0.05$ compared with the EA group.

3.2. Mucosal $m R N A$ Expression of $\alpha 7 n A C h R$ and $N F-\kappa B p 65$. The N-type cholinergic receptor $(\alpha 7 \mathrm{nAChR})$ is the most studied receptor in the cholinergic anti-inflammatory pathway, and nuclear factor $-\kappa \mathrm{B}(\mathrm{NF}-\kappa \mathrm{B})$ is the most important intracellular signal transduction pathway after $\alpha 7 \mathrm{nAChR}$ is activated. Our RT-PCR results showed that intestine mucosal expression of $\alpha 7 \mathrm{nAChR}$ mRNA in EA and PNU group was significantly strengthened compared with that in the I/R group, while in the $\alpha$-BGT group, the difference was not that obvious. When compared with the EA group, $\alpha 7 \mathrm{nAChR}$ mRNA expression in the $\alpha$-BGT group was significantly lower (Figure 1).

The level of NF- $\kappa$ Bp65 mRNA in intestine mucosa was remarkably elevated when suffering from I/R injury $(p<$ 0.05). After EA or PNU282987 is applied, the NF- $\kappa$ Bp65 mRNA expression was significantly decreased compared with the I/R group; nevertheless, the reduction was not so obvious in the $\alpha$-BGT group (Figure 2).

3.3. Serum Inflammatory Factors Expression. After intestinal I/R injury, serum levels of IL- 6 and TNF- $\alpha$ were significantly increased compared with the control group $(154.3 \pm 14.4$ versus $48.3 \pm 16.0$ for IL- 6 and $55.8 \pm 13.4$ versus $18.3 \pm 8.8$ for TNF- $\alpha$, resp.). Their concentrations were significantly downregulated in both EA and PNU groups $(p<0.01)$. Besides, the level of TNF- $\alpha$ in the $\alpha$-BGT group was much lower than in the I/R group $(p=0.026)$ and was higher than that in the EA group ( $p=0.044)$. Nevertheless, serum IL-6 in the $\alpha$-BGT group was much higher than in the EA group ( $p=$ 0.002 ) but was not significantly different from that in the I/R group (Figures 3 and 4).

\section{Discussion}

The immunoregulation and anti-inflammatory effect of acupuncture therapy have been widely recognized. However, the understanding of its structure, function, and mechanism is not yet in depth. In recent years, activation of the nerveimmune regulation of the cholinergic anti-inflammatory pathway has gradually become a hotspot. In this theory, the therapeutic effects of acupuncture on internal organs may be mediated by vagal modulation of inflammatory responses [3]. That is, acupuncture can stimulate the vagus nerve and inhibit proinflammatory cytokines expression via interaction of neurotransmitters, acetylcholine, and $\alpha 7 \mathrm{nAChR}$ subunit on reticuloendothelial macrophage cells [4]. 

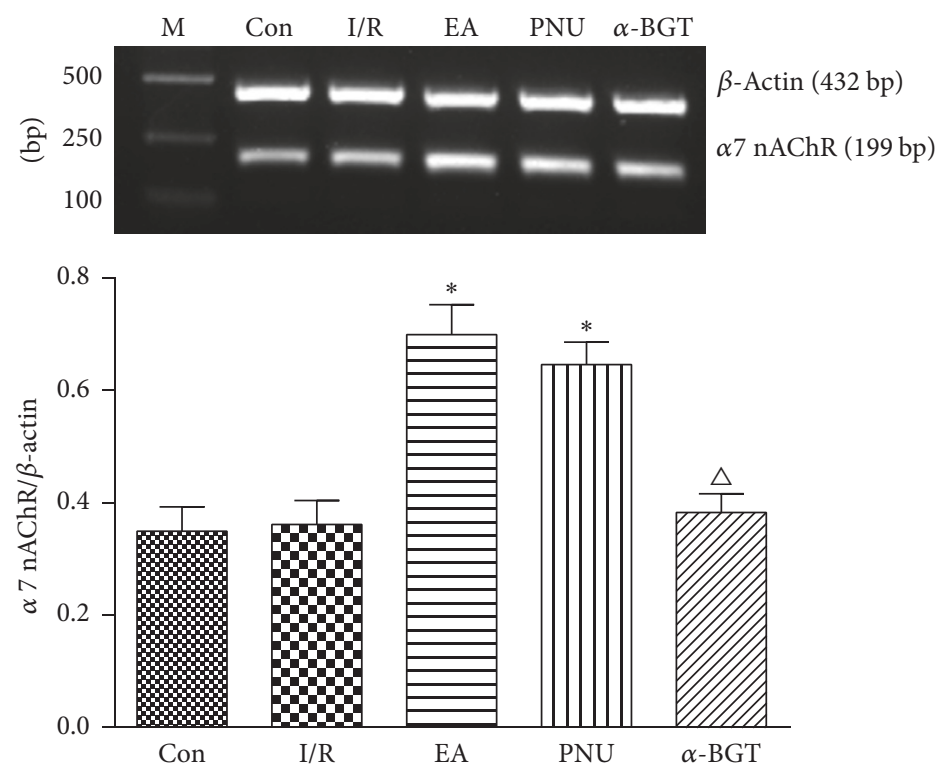

FiguRE 1: Mucosal $\alpha 7 \mathrm{nAChR}$ mRNA expression (blots and relative expressions) assessed by RT-PCR in each group. In the I/R group, mucosal concentration of $\alpha 7 \mathrm{nAChR}$ mRNA was similar to the control group and was significantly strengthened when applied with EA or PNU. In the $\alpha$-BGT group, $\alpha 7 \mathrm{nAChR}$ mRNA level was much lower than in the EA group. M: marker; Con: control group; I/R: I/R group; EA: electroacupuncture group; PNU: PNU282987 group; $\alpha$-BGT: $\alpha$-bungarotoxin group; the abbreviations in Figure 2 are the same $\left({ }^{*} p<0.05\right.$ compared with the I/R group; ${ }^{\triangle} p<0.05$ compared with the EA group).
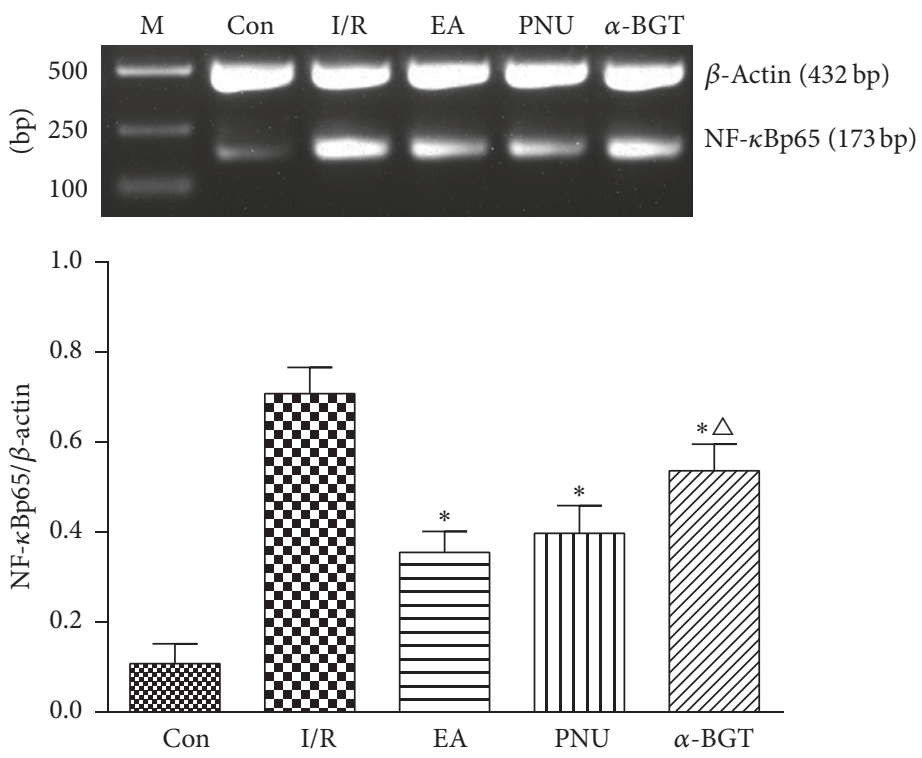

Figure 2: Mucosal NF- $\kappa$ Bp65 mRNA expression (blots and relative expressions) assessed by RT-PCR in each group. Mucosal level of NF$\kappa$ Bp65 mRNA was significantly increased after I/R injury. After EA or PNU282987 was applied, the NF- $\kappa$ Bp65 mRNA level was significantly decreased compared with the I/R group. Its concentration in the $\alpha$-BGT group was higher than that in the EA group $\left({ }^{*} p<0.05\right.$ compared with the I/R group; ${ }^{\wedge} p<0.05$ compared with the EA group).

Previous studies have shown that acupuncture at Zusanli could attenuate the systemic inflammatory response, protect intestinal barrier integrity, and improve organ function and survival rate in hemorrhaged rats [4]. EA at Zusanli also has the potential to reduce serum TNF level in septic rats, protect intestinal barrier integrity from ischemia injury, and reduce postoperative local inflammatory response to alleviate adhesion formation in rats [5-9]. Moreover, abdominal vagotomy or $\alpha 7 \mathrm{nAChR}$ inhibitor could reverse these protective effects of EA [4-9]. PNU-282987 is a selective $\alpha 7 \mathrm{nAChR}$ agonist. A previous study showed that pretreatment with PNU-282987 could prevent NF- $\kappa$ B activation and suppress 


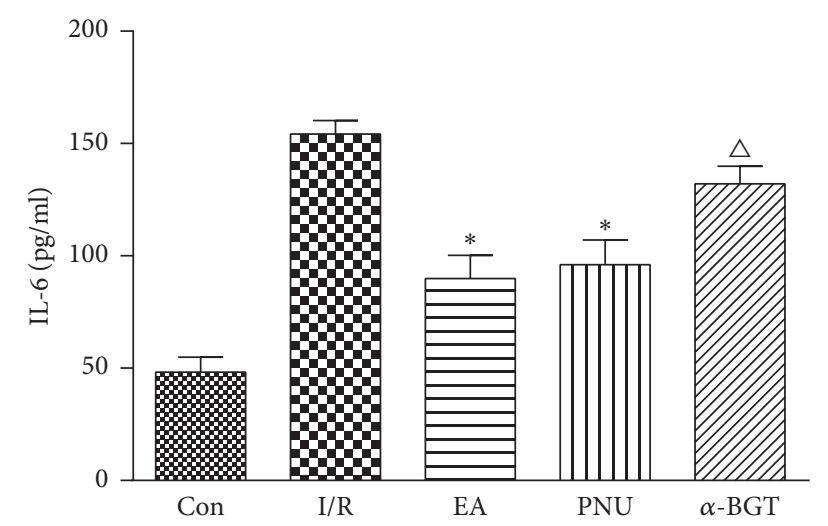

FIgURE 3: Serum IL-6 concentration in each group. Serum IL-6 concentration was significantly increased after I/R injury and was remarkably decreased in both EA and PNU groups when compared with the I/R group. In the $\alpha$-BGT group, IL- 6 level was higher than that in the EA group and showed no difference with the I/R group $\left({ }^{*} p<0.05\right.$ compared with the I/R group; ${ }^{\triangle} p<0.05$ compared with the EA group).

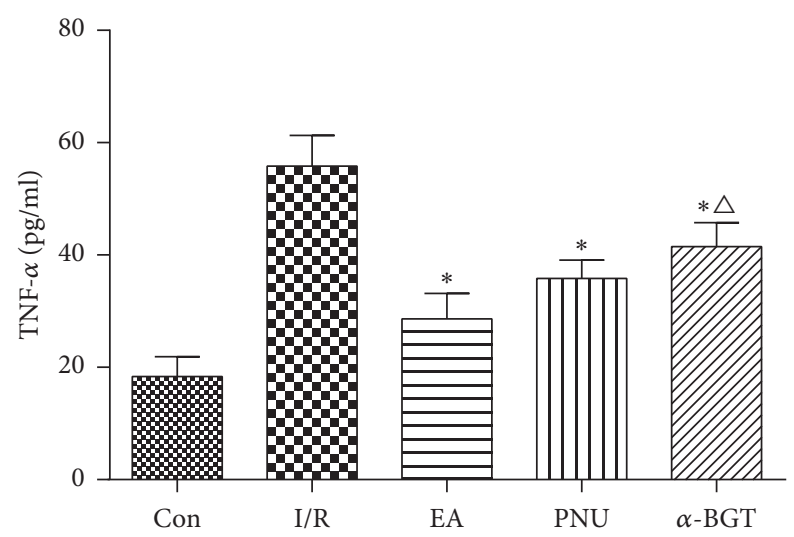

FIGURE 4: Serum TNF- $\alpha$ concentration in each group. Serum TNF$\alpha$ level was greatly increased after I/R attack. Its concentration was significantly decreased in the three treatment groups compared with the I/R group. IL- 6 level in the $\alpha$-BGT group was higher than in the EA group $\left({ }^{*} p<0.05\right.$ compared with the I/R group; ${ }^{\triangle} p<0.05$ compared with the EA group).

cytokine production after hepatic I/R injury in mice [10]. Our study also showed that PNU-282987 and EA have a similar effect in the inhibition of NF- $\kappa$ B and cytokine expression after intestinal I/R injury in rats. These findings indicate that vagal nerve integrity and $\alpha 7 \mathrm{nAChR}$ subunit play an important role in its anti-inflammatory and organ protective effects.

$\mathrm{NF}-\kappa \mathrm{B}$ is a common pathway regulating the transcription of various inflammatory factors. It plays a central role in sepsis and autoimmune diseases. In resting state, NF- $\kappa \mathrm{B}$ binds to NF- $\kappa \mathrm{B}$ inhibitory protein ( $\mathrm{I} \mathrm{B}$ ) to form a trimer complex. When the cells are stimulated by external factors (such as cytokines and mediators), activated $\mathrm{I} \kappa \mathrm{B}$ kinase causes ubiquitination and degradation of $\mathrm{I} \kappa \mathrm{B}$, thereby releasing NF- $\kappa \mathrm{B}$ from the trimer complex and transferring it to the nucleus to activate the transcription process. NF- $\kappa \mathrm{B}$ activation could lead to the expression of many genes, and its overactivation will inevitably cause a series of pathological reactions. Qin et al. reported that EA could suppress the activity of NF- $\kappa$ B signaling pathway to ameliorate inflammatory injury in cerebral $\mathrm{I} / \mathrm{R}$ rats [11]. It is also believed that NF- $\kappa \mathrm{B}$ is one of the main intracellular pathways after $\alpha 7 \mathrm{nAChR}$ activation, which can inhibit the phosphorylation of $\mathrm{I} \kappa \mathrm{B}$ and nuclear translocation of NF- $\kappa \mathrm{B}$ and ultimately suppress proinflammatory cytokine production induced by endotoxin and trauma [12]. Studies have shown that nicotine can inhibit the lipopolysaccharideinduced $\mathrm{I} \kappa \mathrm{B}$ degradation and $\mathrm{NF}-\kappa \mathrm{B}$ nuclear translocation, thereby preventing TNF- $\alpha$ and HMBG1 production [13]. In our research, the content of NF- $\kappa$ B mRNA in intestinal mucosa was significantly increased after I/R injury, and this increase was obviously alleviated after EA or $\alpha 7 \mathrm{nAChR}$ agonist was applied, which was consistent with previous findings.

Acupuncture is a convenient and effective method for gastrointestinal dysfunction diseases [14]. Electroacupuncture is a combination of acupuncture practices and electrophysiological effects. It can increase the needling sensation, produce stronger stimulation intensity, and reduce the work of the twirling needle and is easier to repeat than conventional acupuncture. Hence, it is now widely used in both research and clinical settings. The waveform of EA we performed in this study is condensation-rarefaction wave which is comprised of continuous alternation of condensation wave and rarefaction wave. It is not easy to produce adaption unlike single-pulse wave, and it is often used in situations attempting to increase metabolism, promote blood circulation, and eliminate inflammatory edema. Torres-Rosas et al. even found that the anti-inflammatory effect of EA at ST36 presents a voltage-dependent manner in lipopolysaccharide treated mice [15]. Grech et al. suggest that low-frequency EA can improve immune and stress responses to surgery in anesthetized patients [16]. Future studies can focus on whether different frequencies or voltages have different antiinflammatory effects.

In a previous study, we have confirmed the antiinflammatory and mucosal barrier protection effect of EA at Zusanli during intestinal I/R injury [1]. This study just attempts to explore its possible mechanism from the perspective of cholinergic anti-inflammatory pathway, and the results showed that both EA and $\alpha 7 \mathrm{nAChR}$ agonists could relieve the intestine damage and decrease levels of mucosal NF- $\kappa$ Bp 65 and serum IL- 6 and TNF- $\alpha$. Intraperitoneal administration of $\alpha 7 \mathrm{nAChR}$ antagonist significantly eliminated the anti-inflammatory effect of EA. These observations suggest that EA at Zusanli could protect the intestine from I/R injury possibly by activating the cholinergic antiinflammatory pathway through $\alpha 7 \mathrm{nAChR}$ subunit and NF$\kappa \mathrm{B}$ intracellular signal transduction mechanism. Recently, Dhawan et al. showed that M-type cholinergic receptor $(\mathrm{mAChR})$ also provides protective effects against colitis in in vitro experiments and that $\mathrm{mAChR}$ stimulation might block the phosphorylation of myosin light chain, inhibit myosin contraction, and thus maintain the tight junction protein in a close state and protect the epithelium barrier function $[17,18]$. Therefore, more researches on this mechanism are needed to 
provide a complete theoretical basis for clinical application of Chinese medicine in intestinal ischemic situations.

\section{Conflicts of Interest}

The authors declare that there are no conflicts of interest regarding the publication of this paper.

\section{References}

[1] Y. Geng, D. Chen, J. Zhou et al., "Synergistic effects of electroacupuncture and mesenchymal stem cells on intestinal ischemia/reperfusion injury in rats," Inflammation, vol. 39, no. 4, pp. 1414-1420, 2016.

[2] H. Wang, L. Wang, X. Shi et al., "Electroacupuncture at zusanli prevents severe scalds-induced gut ischemia and paralysis by activating the cholinergic pathway," Evidence-Based Complementary and Alternative Medicine, vol. 2015, Article ID 787393, 6 pages, 2015.

[3] H.-D. Lim, M.-H. Kim, C.-Y. Lee, and U. Namgung, "Antiinflammatory effects of acupuncture stimulation via the vagus nerve," PLoS ONE, vol. 11, no. 3, article e0151882, 2016.

[4] M.-H. Du, H.-M. Luo, S. Hu, Y. Lv, Z.-L. Lin, and L. $\mathrm{Ma}$, "Electroacupuncture improves gut barrier dysfunction in prolonged hemorrhagic shock rats through vagus antiinflammatory mechanism," World Journal of Gastroenterology, vol. 19, no. 36, pp. 5988-5999, 2013.

[5] X.-M. Song, J.-G. Li, Y.-L. Wang et al., “The protective effect of the cholinergic anti-inflammatory pathway against septic shock in rats," Shock, vol. 30, no. 4, pp. 468-472, 2008.

[6] A. Villegas-Bastida, R. Torres-Rosas, L. A. Arriaga-Pizano, J. Flores-Estrada, A. Gustavo-Acosta, and M. A. Moreno-Eutimio, "Electrical stimulation at the ST36 acupoint protects against sepsis lethality and reduces serum TNF levels through vagus nerve- and catecholamine-dependent mechanisms," EvidenceBased Complementary and Alternative Medicine, vol. 2014, Article ID 451674, 8 pages, 2014.

[7] S. Hu, M.-H. Du, H.-M. Luo et al., "Electroacupuncture at Zusanli (ST36) prevents intestinal barrier and remote organ dysfunction following gut ischemia through activating the cholinergic anti-inflammatory-dependent mechanism," Evidence-Based Complementary and Alternative Medicine, vol. 2013, Article ID 592127, 10 pages, 2013.

[8] M.-H. Du, H.-M. Luo, Y.-J. Tian et al., "Electroacupuncture ST36 prevents postoperative intra-abdominal adhesions formation," Journal of Surgical Research, vol. 195, no. 1, pp. 89-98, 2015.

[9] L. Zhang, H. Wang, Z. Huang et al., "Inhibiting effect of electroacupuncture at Zusanli on early inflammatory factor levels formed by postoperative abdominal adhesions," Evidence-Based Complementary and Alternative Medicine, vol. 2014, Article ID 950326, 5 pages, 2014.

[10] F. Li, Z. Chen, Q. Pan et al., "The protective effect of PNU282987, a selective $\alpha 7$ nicotinic acetylcholine receptor agonist, on the hepatic ischemia-reperfusion injury is associated with the inhibition of high-mobility group box 1 protein expression and nuclear factor $\kappa$ b activation in mice," Shock, vol. 39, no. 2, pp. 197-203, 2013.

[11] W.-Y. Qin, Y. Luo, L. Chen et al., "Electroacupuncture could regulate the NF- $\kappa$ B signaling pathway to ameliorate the inflammatory injury in focal cerebral ischemia/reperfusion model rats," Evidence-Based Complementary and Alternative Medicine, vol. 2013, Article ID 924541, 15 pages, 2013.
[12] W. J. de Jonge and L. Ulloa, "The alpha7 nicotinic acetylcholine receptor as a pharmacological target for inflammation," British Journal of Pharmacology, vol. 151, no. 7, pp. 915-929, 2007.

[13] R. W. Saeed, S. Varma, T. Peng-Nemeroff et al., "Cholinergic stimulation blocks endothelial cell activation and leukocyte recruitment during inflammation," The Journal of Experimental Medicine, vol. 201, no. 7, pp. 1113-1123, 2005.

[14] H. Li, T. He, Q. Xu et al., "Acupuncture and regulation of gastrointestinal function," World Journal of Gastroenterology, vol. 21, no. 27, pp. 8304-8313, 2015.

[15] R. Torres-Rosas, G. Yehia, G. Pena, P. Mishra, M. Del Rocio Thompson-Bonilla, and M. A. Moreno-Eutimio, "Dopamine mediates vagal modulation of the immune system by electroacupuncture," Nature Medicine, vol. 20, no. 3, pp. 291-295, 2014.

[16] D. Grech, Z. Li, P. Morcillo et al., "Intraoperative low-frequency electroacupuncture under general anesthesia improves postoperative recovery in a randomized trial," JAMS Journal of Acupuncture and Meridian Studies, vol. 9, no. 5, pp. 234-241, 2016.

[17] S. Dhawan, I. H. Hiemstra, C. Verseijden et al., "Cholinergic receptor activation on epithelia protects against cytokineinduced barrier dysfunction," Acta Physiologica, vol. 213, no. 4, pp. 846-859, 2015.

[18] K. E. Cunningham and J. R. Turner, "Myosin light chain kinase: pulling the strings of epithelial tight junction function," Annals of the New York Academy of Sciences, vol. 1258, no. 1, pp. 34-42, 2012. 


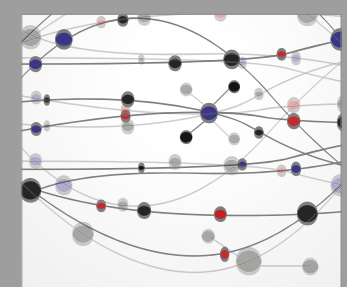

The Scientific World Journal
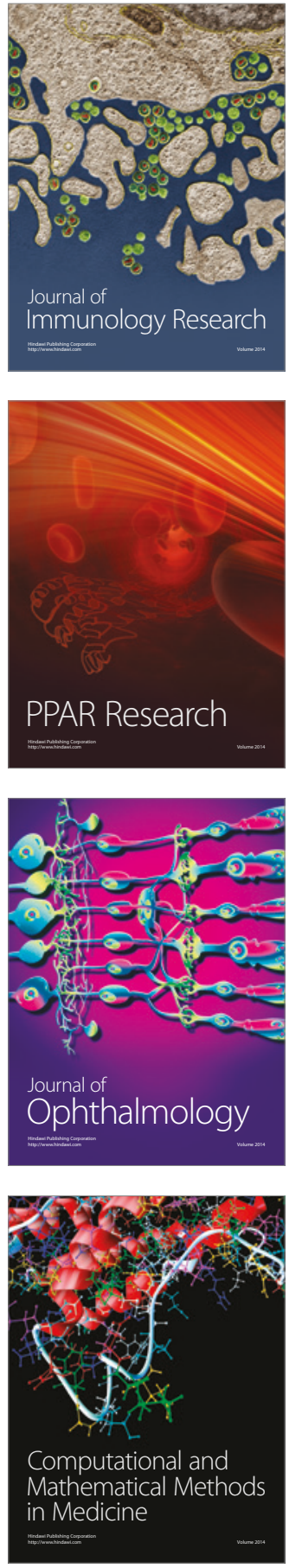

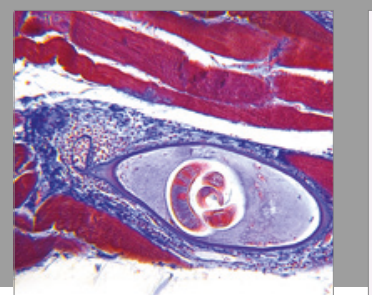

Gastroenterology Research and Practice
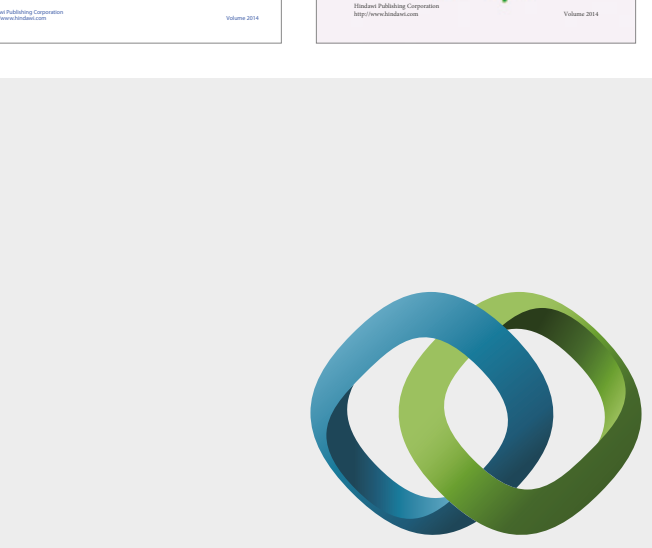

\section{Hindawi}

Submit your manuscripts at

https://www.hindawi.com
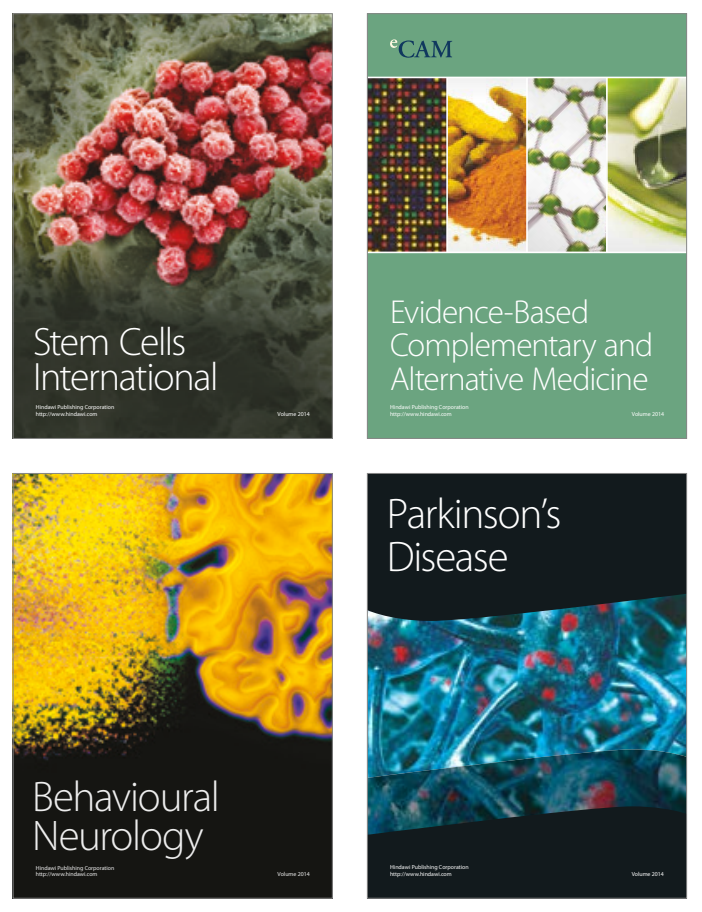
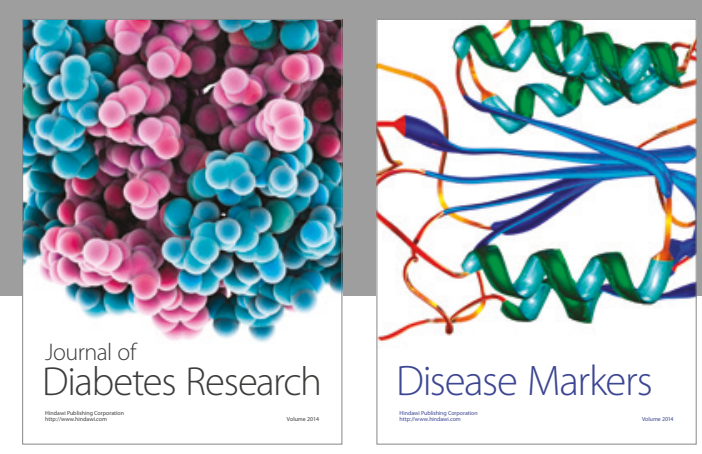

Disease Markers
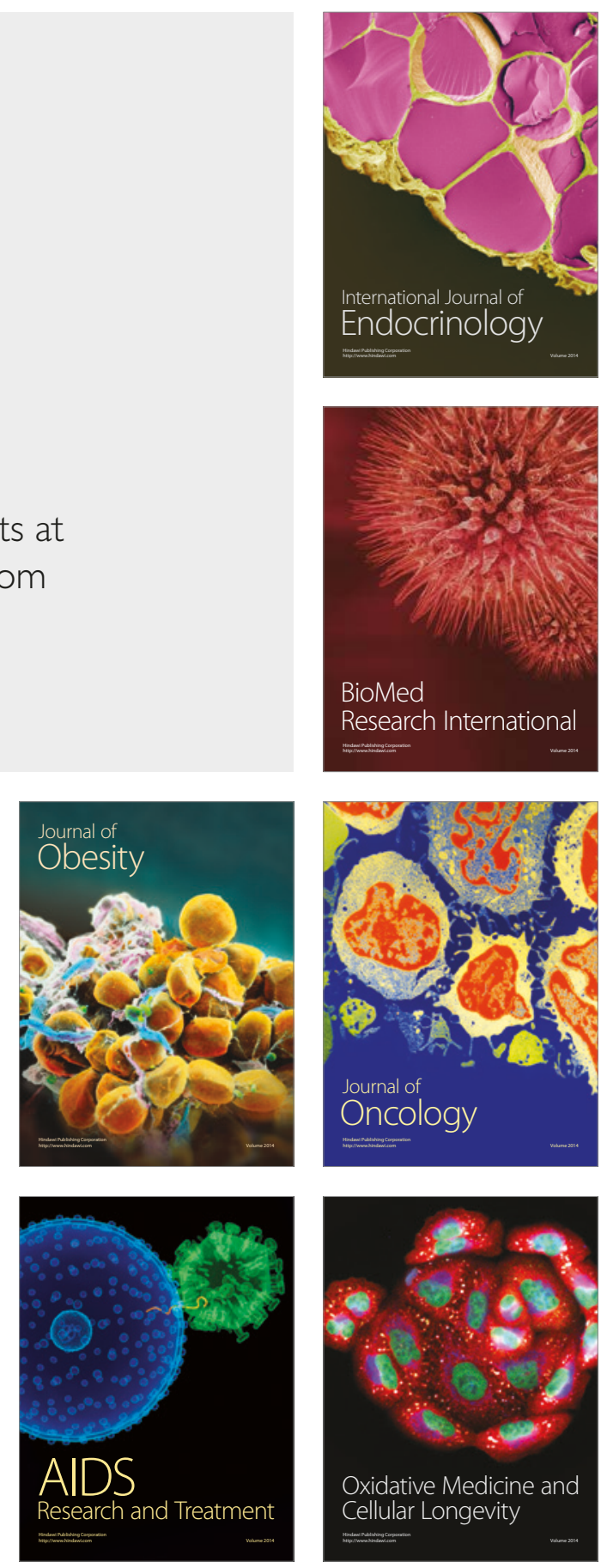